\title{
GLOBAL RESEARCH DECENTRALIZED AutonOMOUS ORGANIZATION (GR-DAO): A DAO OF GLOBAL RESEARCHERS
}

\author{
Kelly L. Page ${ }^{1}$ and Adel Elmessiry ${ }^{2}$ \\ ${ }^{1}$ LWYL Studio, Chicago, IL, USA \\ ${ }^{2}$ AlphaFin, Nashville, TN, USA
}

\begin{abstract}
The latest trend in Blockchain formation is to utilize decentralized autonomous organizations $(D A O)$ in many verticals. To date, little attention has been given to address the global research domain due to the difficulty in creating a comprehensive framework that can marry the cutting edge of academic grade scientific research with a decentralized governance body of researchers. A global research decentralized autonomous organization (GR-DAO) would have a profound impact on the research community academically, commercially, and the public good.

In this paper, we propose the GR-DAO as a global community of researchers committed to collectively creating knowledge and sharing it with the world. Scientific research is the means for knowledge creation and learning.
\end{abstract}

The GR-DAO provides the guidance, community and technological solutions for the evolution of a global research infrastructure and environment. Through its design, the GR-DAO embraces, enhances and extends the model of research, research on decentralization and DAO as a model for decentralised and autonomous organizing. This design, in turn, improves most of the uses for and applications of research for the greater good of society.

The paper examines the core motivation, purpose and design of the GR-DAO, its strategy to embrace, enhance and extend the research ecosystem, and the GR-DAO design uses across the DAO ecosystem.

\section{KEYWORDS}

Scientific Research, Researcher, Research, Knowledge, Learning, Cocreated Knowledge, Applied Research, Decentralized Autonomous, Organization, DAO, Research Model, Research Activity, Blockchain, Emerging Technology, Incentive Design, Reputation Staking, Distributed Ledger Technology, Decentralized Infrastructure.

\section{INTRODUCTION}

While much scientific research is publicly funded or in the public interest, it is not available to the public. This is a global problem fueled by the increasing centralization of scientific research and knowledge in our academies and foundations; and the private commodification of research activities and outputs.

David C. Wyld et al. (Eds): COMIT, CRBL, BIOM, WiMNeT, SIP, AISO - 2021

pp. 77-90, 2021. CS \& IT - CSCP 2021

DOI: $10.5121 / \mathrm{csit} .2021 .111708$ 
Now imagine a different world. A world where cutting edge scientific research is publicly owned and available to everyone. Where researchers are paid fairly, equitably, and transparently for their contributions to science, their participation in the global research community, and the impact and attribution of their research is effectively and efficiently tracked and remunerated over time, and openly in a distributed digital ledger.

Let's take this world even further. You are an early career researcher working on a cutting edge research idea. You live and work in Kandahar, Afghanistan. You identify as female with three children and English is not your first language. Everyday you too go to work to do scientific research, contributing to a global research community for the public good. You too are a cutting edge scientific researcher and your contribution is as equally valued and remunerated in the global research community as that of your peer and collaborator, who lives in Boston, and is tenured at a world leading research institution.

The creation of these research worlds to address the shortcomings of the current organizational design of scientific research, especially its centralized and private commodification, is socially, financially and technically possible. The latest trend in Blockchain formation is to utilize decentralized autonomous organizations (DAO) in many verticals. To date, little attention has been given to address the global research domain and its design due to the difficulty in creating a comprehensive framework that can marry the cutting edge of academic grade scientific research with a decentralized governance body of researchers.

To address this, we propose the creation of a Global Research Decentralized Autonomous Organization (GR-DAO). A GR-DAO would have a profound impact on the research community both academically, commercially and for the public good. Decentralized Autonomous Organizations (DAO) represent the next evolution in global organizational governance. They are on the rise, and it is an exciting time for research scholars globally and organizational and technology scholars in particular, to address this emerging phenomenon with new theory and solid empirical research for a global research community.

The paper examines the motivation, purpose and design of the GR-DAO, its strategy to embrace, enhance and extend the research ecosystem, and the GR-DAO design uses across the DAO ecosystem.

\section{Motivation}

We are experiencing the rise in the private commodification and centralization of scientific and/or academic research. These two phenomena have contributed to a number of challenges for the global research community. The most pressing challenge being that while much scientific research is publicly funded or in the public interest, it is not available to the public. The creation of a Global Research Decentralized Autonomous Organization (GR-DAO), would have a profound impact on the research community both academically, commercially and for the public good.

In this section we discuss the two driving motivations for the establishment of a GR-DAO including the rise in: 1) Research Commodification and 2) Research Centralization.

\subsection{Research commodification}

Research is any creative systematic activity undertaken in order to increase the stock of knowledge, including knowledge of humankind, culture and society, and the use of this knowledge to devise new applications [1]. It is an activity motivated for 1) the public interest, that 
is for the betterment of society and the advancement of knowledge for all humankind [2][3] and/or 2) commercial interest and application, that is advancing self or an entity for interest, compensation or commercial gain [4][5].

To serve both motives, two complementary forms of research activity have emerged in our modern culture [4]: Scientific which is also called basic or academic (or of the academy) and Applied or professional research designed for practical purposes and commercial advancement. The two differ in terms of methodology, methods and the interests of the sponsoring parties.

Scientific research is more often motivated for academic or general interests of the public, is performed by researchers in research institutions applying systematic and constructed scientific methods and/or protocols to obtain, analyze, and interpret data, where the intention is to identify facts and/or opinions that will assist in solving the problem or dealing with the situation [6][7]. It is also academic or of the academy, in this it is a core activity conducted or supported by members of public and private, largely non profit universities, and funded by public tax money, foundations or donors. Applied research in contract is designed to answer specific questions aimed at solving practical or use problems, be they technical, social or for commercial purposes. Conducted by private entities, both nonprofit and for profit, it is more often funded privately and conducted for proprietary purposes[6][7].

The purpose of these forms of research may serve to advance knowledge, the devising of new applications and for the betterment of society. Yet, while we expect the commodification of applied research as a 'private good', since the 1980's, we are increasingly seeing the rise of the commodification of publicly funded scientific research, and shift from a public good to a private one that can be traded [8].

A complex phenomenon, commodification is identified with commercialization, that is, the pursuit of profit by academic or media institutions through selling the expertise of researchers and the results of their inquiries [9]. The commodification of scientific research is part of a comprehensive and long-term social development [9]. This development is often described as the economization, or economic instrumentalization, of human activities and institutions, or even entire social subsystems [10][11]. In the higher education sector, we further see this activity through the lens of the marketization of higher education - the growing influence of market forces on higher education, resulting in what is defined by Fairclough (1993) as the marketization of academic discourse [12][13].

Commodification implies the expropriation of goods from the particular communities that produced them by reducing the intrinsic, community value of these goods to their pecuniary exchange value on an independent market [14]. Increasingly we are seeing the rise in higher education and its activities, moving from a once held belief of it as a public good of benefit to the individual and to the public, to it being a private commodity for sale.

As a private commodity for sale it benefits the interest of the few and can take many forms such as research that is commercially funded, strategic research alliances or partnerships with private firms, or interest groups, paid or sponsored positions such as research or endowed Chairs, as well as the commercial activities of many of the major scientometric databases and media companies with a vested interest. The acquisition and exploitation of intellectual property and patents on the results of scientific research is another form [9].

This rise in the private commodification of scientific research as a private good for sale contributes to a number of challenges for a global research community committed to research as a public good, in the public's interest and the public domain. 


\subsection{Research Centralization}

The organization of research and research cultures is becoming increasingly centralized, hierarchical and top-down as well as privatised. This is in contrast to over twenty or thirty years ago, when many research and higher education institutions were relatively decentralised, flatter and with more autonomy at the department, school or faculty level and in research activity. [15][16][17].

The centralization of scientific research includes: the central organization of institutional research activity, research evaluation and funding, publishing, as well as a centralist view of the global research community.

- Institutional Research Activity: The organization of institutional research activity is becoming increasingly centralised such as in the creation of administrative units centrally to oversee research policy and activities; consolidation and marketization of research training programs for the education of early career researchers; central decision making as to what and whom to fund and support, and research activity evaluation.

- Research Evaluation/Funding: This is coupled with the formalization of bureaucratic research bodies for the conduct of research assessment exercises (e.g., REF in the UK) in order to receive public funding, centralising the decision as to what research is of value to the public good (money) and the indicators upon which this research is evaluated.

- Research Publishing: We've experienced the rise in private centralization of essential research activities such as the peer-review, publishing and the dissemination process, today called the 'academic publishing industry'. Once overseen by researchers and institutions themselves, it is today a robust business sector that economically benefits greatly from researchers and the public. Worldwide it has sales amounting to more than USD 19 billion. This positions it between the music industry and the film industry in revenue [25]. Yet, it is a closed system with high barriers to entry and limited public access. It is dominated by five large publishing houses: Elsevier, Black \& Wiley, Taylor $\&$ Francis, Springer Nature and SAGE, which control more than $50 \%$ of the market between them. In its current business model, public funds fund all stages of research production, the research faculty who manage the peer-review process for free and esteem, and then pays through an institution again to have member access to the research articles and results archived behind a paywall.

- Research Community: There is an inherent centralism in the view of participation in research in the global research community. The community is dominated by and centralised from a western, developed and euro-centric world-view in knowledge creation, value and its dissemination. For example, often science is not not encouraged in developing countries because it is expensive, yet it is of inherent value and impact to their development and progress, as much if not more as developed ones. Further, researchers in these countries do not have the same level or type of support for their contribution.

This rise in the centralization of scientific research is in contrast to what is stressed in much management and organization research as to what fosters cultures of innovation and sharing-- all critical ingredients for scientific research [18][19][20].

\subsection{Emerging Challenges}

The rising centralisation of scientific research, coupled with its private commodification, is contributing to the challenges for a global research community to do research for the public and 
in the global domain (Table 1). These include (but are not limited to): Unsustainable business model, funding is inflexible and inefficient, rising inequity, fixed boundaries and social norms, Inaccessibility, Poor Transparency.

Table 1. Current Challenges of Our Global Research Community

\begin{tabular}{|c|c|}
\hline Challenge & Description \\
\hline $\begin{array}{l}\text { Unsustainable } \\
\text { Business } \\
\text { Model }\end{array}$ & $\begin{array}{l}\text { The labor of research business model is not economically workable or } \\
\text { sustainable for researchers or institutions participating in the knowledge } \\
\text { creation economy. } \\
\text { Researchers can not sustain the work product or load expected of them, esp. } \\
\text { with a rise in 'work for hire' doctrines resulting in limited to no long term } \\
\text { fiscal benefit for the creative work contributed. }\end{array}$ \\
\hline $\begin{array}{l}\text { Funding is } \\
\text { Inflexible and } \\
\text { Inefficient }\end{array}$ & $\begin{array}{l}\text { - How, who and when is research funded, who makes these decisions and the } \\
\text { evaluation criteria upon which they are made are not transparent. } \\
\text { - Science funding is a mess with academic researchers having to rely on outside } \\
\text { grants in order to pay salaries and buy their equipment. } \\
\text { This results in many leading researchers spending some } 40-50 \% \text { of their time } \\
\text { writing research grants and responding to grant administration and evaluation } \\
\text { processes. }\end{array}$ \\
\hline $\begin{array}{l}\text { Rising } \\
\text { Inequity }\end{array}$ & $\begin{array}{l}\text { - Researchers are not paid equitably or enough for their labor, skills and } \\
\text { contribution to the knowledge and research economy. } \\
\text { The rising inequality continues to disadvantaged people of color, women and } \\
\text { those of less economic means are sidelined or not given access to research } \\
\text { training, research and denied due attribution for their research labor and work. }\end{array}$ \\
\hline $\begin{array}{l}\text { Fixed } \\
\text { Boundaries } \\
\text { and } \\
\text { Social Norms }\end{array}$ & $\begin{array}{l}\text { - The culture is fixed with boundaries and high barriers to entry. It is of benefit } \\
\text { to only those who have access to a "certain" education or the institutions which } \\
\text { are deemed "worthy" or "elite" in research terms to participate. } \\
\text { The current structure de-incentivises interdisciplinary research across } \\
\text { boundaries, and/or researchers playing at the edges or outside the community } \\
\text { norms. }\end{array}$ \\
\hline \multirow[t]{2}{*}{ Inaccessibility } & $\begin{array}{l}\text { - Scientific research work is not in the public domain. It is archived behind a } \\
\text { paywall and only accessible to those who can afford to pay membership fees or } \\
\text { have access through institution affiliation. }\end{array}$ \\
\hline & $\begin{array}{l}\text { - Those research works which are publicly accessible, are often not valued } \\
\text { or ranked as worthy for the promotional / tenure system universities put in } \\
\text { place to evaluate the research deliverables of a researcher. }\end{array}$ \\
\hline $\begin{array}{l}\text { Poor } \\
\text { Transparency }\end{array}$ & $\begin{array}{l}\text { The agreements and contracts between researchers and institutions, and } \\
\text { private interests are not transparent. It is difficult to know who is funding } \\
\text { research, who owns it and who has the rights of access to it. } \\
\text { There is also limited transparency in how much the industry or sector is } \\
\text { actually worth or of value and the impact or access to that value in the } \\
\text { public domain. }\end{array}$ \\
\hline
\end{tabular}

In short, while much scientific research is publicly funded or in the public interest, it is not available to the public for advancing the knowledge of humankind. 


\section{The Proposed Solution}

To address the shortcomings of the current organizational design of scientific research, especially its centralized and private commodification, we propose the creation of a Global Research Decentralized Autonomous Organization (GR-DAO).

A DAO is a non-hierarchical organizations that perform and record routine tasks on a peer-topeer, cryptographically secure, public network, and rely on the voluntary contributions of their internal stakeholders to operate, manage, and evolve the organization through a democratic consultation and voting process [26][27]. DAOs coordinate routine tasks through cryptographic routines (as opposed to human routines). Blockchain-based organizing and the resulting DAOs have the ability to replace centralized intermediaries in other applications requiring complex coordination [29]. Blockchain has the clear potential to substantially upgrade the processes and organization traditionally underpinning academic science [35].

\subsection{Blockchain Applications to Scientific Publishing}

The goal of blockchain applications for scientific research has been to establish origins of research outputs, and tracking how the assets change through the publishing lifecycle. A summary of applications are summarised in Table 2.

Table 2. Blockchain-based Scientific Publishing Applications

\begin{tabular}{|c|c|}
\hline Application & Description \\
\hline ARTIFACTS & $\begin{array}{l}\text { - Records immutable chains of scholarly artifacts (e.g., figures, images, etc.) to } \\
\text { establish attribution and proof-of-existence of early scientific work. } \\
\text { Focuses on research asset creation, tracking and sharing of publishing } \\
\text { processes. }\end{array}$ \\
\hline Manubot & - Manuscript version control needed during the editing and publishing process. \\
\hline Orvium & $\begin{array}{l}\text { - Focuses on integrating blockchain technology into the publication life cycle } \\
\text { while also encouraging open-science and research dissemination aims. } \\
\text { Ability to create Decentralized Autonomous Journals (DAJs) with their own } \\
\text { governance rules and licensing and subscription models. }\end{array}$ \\
\hline$\underline{\text { Pluto }}$ & $\begin{array}{l}\text { - Manage transfers of value through the research lifecycle by using smart } \\
\text { contracts and tokens on the blockchain and smart contract environment } \\
\text { Ethereum. } \\
\text { Allows users to submit and store different types of scientific } \\
\text { information/data (with Digital Object Identifiers) and retain copyright } \\
\text { control. }\end{array}$ \\
\hline $\begin{array}{l}\text { Sciencematters } \\
\text { and Eureka }\end{array}$ & $\begin{array}{l}\text { Open-access web-based OA publishing platform that focuses on single } \\
\text { observation studies (and also encourages negative and replication } \\
\text { studies); works in tandem with a journal submission and token reward } \\
\text { system powered by blockchain. }\end{array}$ \\
\hline$\underline{\text { Scienceroot }}$ & $\begin{array}{l}\text { - Utilizing its own token [called "Science Token" (ST)], a digital wallet, } \\
\text { and smart contracts operating on a proof-of-stake consensus. } \\
\text { - Decentralized collaboration platform, marketplace, and repository. } \\
\text { - Relies on tokenization to drive the research process. }\end{array}$ \\
\hline
\end{tabular}


Most blockchain applications like those shared in Table 2 focus on blockchain applications for the peer-review, publishing and citation activities of scientific research. This is not comprehensive of the knowledge creation process. The GR-DAO proposal is a community wherein blockchain application and token economy supports and actuates the entire knowledge creation, workflow process and validates the contribution of researchers throughout the knowledge generation experience.

\subsection{DAO Design Benefits}

While DAO as a type of organization is relatively new, researchers in the fields management and organizational design have long documented the numerous social and cultural benefits of more flatter organisational structures, decentralisation of decision making and local autonomy over more structured and centralised ones. This is especially apparent for the fostering of cultures of innovation [18], a culture which is critical for the development of research, its evaluation and dissemination.

A global research community would benefit from being a decentralised organization as it would provide for more effective communication horizontally as well as vertically [19], encourage creativity among and between members [20], generate imaginative solutions to problems [21], increase levels of research motivation and satisfaction [22], as well as increase member responsiveness to changes in the external environment [23]. A detailed meta-analysis of the determinants of organisational innovation further confirm the significant negative influence of centralisation and of formalisation on organisational innovation [24], providing support for a more decentralized organization design.

In the following sections of this paper we outline the social design, legal and economic design of the GR-DAO, as well as its strategy to embrace, enhance and extend the global research ecosystem, as well as research across the DAO ecosystem.

\section{GR-DAO Social Design}

\subsection{About}

The GR-DAO is a global community of researchers committed to collectively creating knowledge and sharing it with the world. Scientific research is the means for knowledge creation and learning.

\subsection{Mission}

The mission of the GR-DAO is to embrace, enhance and extend scientific research in the public interest, as a public good and for advancing the knowledge of humankind publicly.

The GR-DAO will provide the guidance, community and technological solutions for the evolution of a global research infrastructure and community. Through its design, the GR-DAO embraces, enhances and extends the model of research, dedicates resources for scientific research on DAO's as a model for decentralised and autonomous organizing in research and technology communities. Our aim is to improve most of the uses for and applications of scientific research for the greater good of society. 


\subsection{Community Members}

The GR-DAO is a global community of researchers committed to collectively creating knowledge and sharing it with the world. Scientific research is the means for knowledge creation and learning. The community includes (but is not limited to): scientists, researchers, philosophers, engineers, developers, and those with an active participatory interest in the pursuit and sharing of knowledge.

\subsection{Foundational Beliefs and Values}

At the heart we are the 'Founding Mothers', the 'Creators of Intelligence' and the 'Makers of Intelligent Life.' The GR-DAO community shares the following core beliefs as foundational elements to articulate their values:

- We are a collective - means we are a group of individuals who collectively as a community create and share knowledge with the world.

- We nurture the pursuit of knowledge - meaning we support research activities, education and the ways people learn, share and advance understanding and knowledge of the world.

- We hold space for research as a public good - available, accessible, transparent and verifiable.

- We champion equity - meaning equitable community participation and access to knowledge, as well as the equitable distribution of power and decision-making

- We honor individual freedom of choice - meaning an individual's power to choose, be it of research participation or ownership of one's personal information, individual security or privacy.

- We aim for efficiency - meaning the ratio of the useful work performed by a human, machine, or process to the total energy expended.

- We collaborate for the common good - meaning improving power distribution by raising the power of the most individuals without harming the least powerful.

The GR-DAO recognizes that these community values and core beliefs provide cohesion and longevity in the GR-DAO and in decentralized systems at large.

\subsection{Community Activities}

The GR-DAO will support research activities such as: research funding, research review and evaluation, research education, as well as the dissemination of scientific research via open access and creative commons licenses in the public domain. At start-up, the GR-DAO will focus on research pertaining to decentralization, DAO, blockchain applications and cryptocurrency, and expand its focus as the community, it's member design and its activities grow.

\subsection{Community Agreements}

Community agreements are a set of basic ground rules or policies that are asked of participants in the community to follow. A living constitution if you will, that is regularly revised and updated with the goal is to create an open and inclusive space so that every individual has the ability to participate, flourish and be heard, as well as to know what is expected of them and how they will exchange value as a member of the community.

To execute the agreement the GR-DAO will use smart contracts to execute the agreement logic in response to events, executing the performance of various tasks, processes or transactions that 
have been programmed into them to respond to a given set of conditions as set-down in the community agreement.

\subsection{Community Development}

The GR-DAO will be committed to the health and well-being of its community and members with ongoing development, education and learning activities. From the on-boarding of new members, to learning about advancements in technology, infrastructure and policies that could have an impact on the GR-DAO as a global research community committed to embracing, enhancing and extending research for the public good.

\section{Gr-Dao Legal Consideration}

One important aspect of creating a successful DAO is to consider the legal structure and wrapper for how it will interact with the external entities. Current research organizations depend on centralized entities for the grant solicitation, allocation and management. This structure is largely inefficient and requires a huge amount of human intervention while creating a single point of failure [30].

In a DAO framework, the DAO member governance is conducted through the decentralized consensus mechanism which is more efficient and much more resilient.

\subsection{Legal Structure}

To be able to operate in both the real world and the virtual space in loosely coupled a structure in both must exist. This is accomplished by creating a legal rapper in the non-DAO world that would function as the representative of the DAO in a jurisdiction allowing it to conduct operations within their jurisdiction and in collaboration with other jurisdictions while maintaining its separate virtual existence. For example an association could be created under Swiss law as a nonprofit with a mandate to be engaged by the DAO so that it carries out three major functions namely: engage in contractual agreements, receive funds and issue appropriate receipts, and provide employment structures for those where it is required.

\subsection{Governance}

In most DAOs Governance is a function allowing the DAO to vote and reach decisions based on the collective will of the DAO. It is an important mechanism to make sure that the function of the DAO is going into the right direction as seen by the collective. An important aspect of the governance is to be able to weigh the difference in votes eternity and figuring out a way to make each vote has some sort of accountability and consequences to the voter.

\subsection{Contribution-Based Internal Administration}

The question that we'll have to answer is how are we going to make those decisions within the DAO itself. One such approach is to use a contribution based system. The contribution system in research there will be correlated with the amount of knowledge each individual individual actually contributes to the DAO. This is a way for each researcher to acquire more contribution tokens as they contribute more to the actual system itself and to the mission of the DAO. When taking a vote on a certain proposal for research or a decision each individual can stick an amount of this gained contribution to emphasize their support for this decision. 
When the decision is voted on the member risks that stick to contribution on the outcome of the collective. Thus it allows the collective to steer towards a common vision for the research being funded, reviewed, conducted or shared with the end of the DAO.

\section{GR-DaO GaMe Theory AND Resilience}

Game theory can be defined as the study of mathematical models of strategic interaction between rational decision-makers [31]. Our governance structure should continue a game theory-like incentive mechanism that allows the decision makers to have incentives for voting in the right direction as set by the collective and to be penalized for misleading the collective.

\subsection{Game-Theoretical Design}

One of the interesting game theory works is established in the cooperation through social influence done by Molinero et. al. (2015) and (2021) [31][32]. The main contribution is to show the relationship between the influence spread phenomenon coming from social network analysis and the binary decision-making in the voting system. We utilize the same basis but replacing the gains with our version of knowledge based reputation.

\subsection{Equal Distribution of Knowledge}

Since the main objective of the DAO is to promote knowledge, it is natural to utilize knowledge as the basis of how the system works. To create an equatibal system we would need to assure the equal distribution of knowledge within the system. That means that access to knowledge is available for everyone. It is the capability of each individual to achieve additional knowledge while contributing to the overall research activities of the system that gains them additional points and power.

\subsection{Attack Resistance}

In any system with value attacks or text or nothing but assured. The system must create a mechanism. It isn't to make the coolest of the attack, it is the attack much higher than the gains acquired by a successful attack. The GR-DAO is no different in that it tries to mitigate those attacks on the overall collective.

\subsubsection{Sock Puppet Attacks}

The 1st mechanism here is to assure that the only way for individuals to gain weight in the system, the system, meaning they are able to affect the decisions of the collective is by contributing to the actual output of the collective. The output is basically research and adding to the knowledge of the collective. The more people contribute the more power they will have to affect the decisions of the collective And since that power is not financially driven it cannot be bought but it can be only acquired.

Finally the requirement of creating a substantial body of work will create a deterrent for the players that want to attack the actual collective because in the event of losing they will lose all the hard work that they have put into the season.

This is akin to the proof of work however in our case we call it proof of research. 


\subsubsection{DOS Attacks}

Denial of service attacks are another way of trying to sabotage a system that is working. The most effective way of defending against a denial of attack is by creating a cost for each request of service. This cost is set so that an entity cannot create an infinite number of requests and as tying up the resources of the collective while effectively shutting down the services.

\section{GR-DAO ECONOMIC DESIGN}

Unlike the traditional DAO models our model will use non-fungible tokens (NFT's) as the base for onboarding members into the collective. A non-fungible token (NFT) is a representation of a unique digital asset, essentially a digital certificate of authenticity, that cannot be equally swapped or traded for another NFT of the same type. They are stored on a blockchain or a distributed ledger and are used to represent ownership of unique items [34].

Each member of the GR-DAO will receive an NFT for their membership with a wallet. The wallet associated with the NFT, and the NFT will work as a reputation holder for the knowledge reputation tokens held for this member. This dual token system allows the user to vote on different proposals in the DAO [33].

The NFT based DAO membership model is depicted in Figure 1.

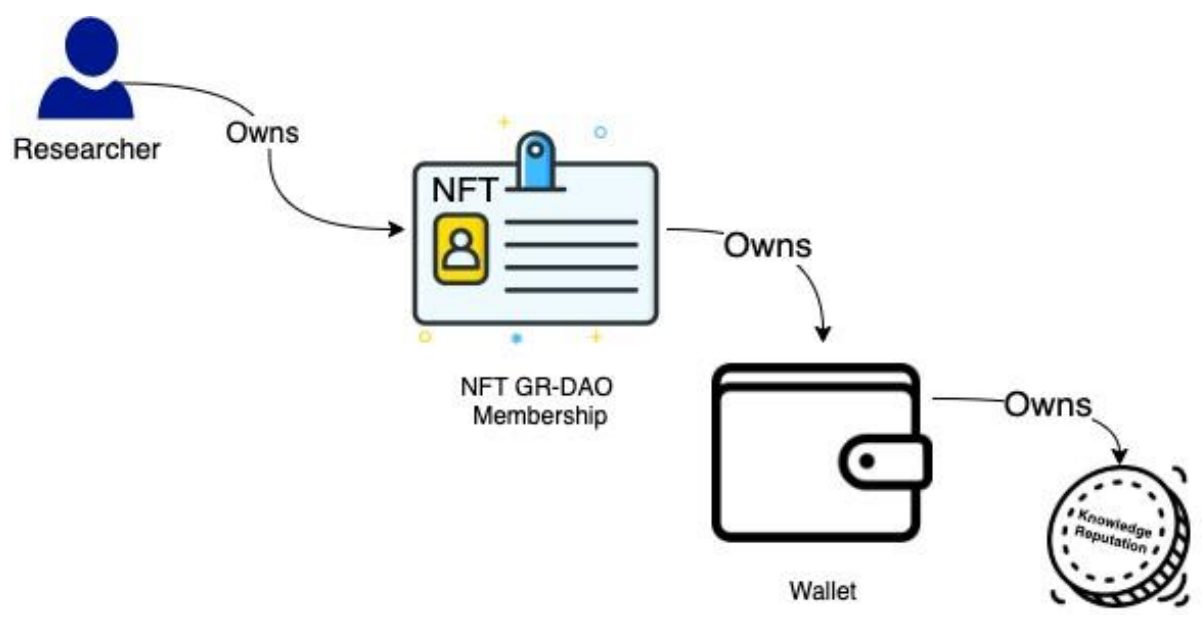

Figure 1. NFT Based DAO Membership Model

\subsection{NFTs Model}

Each of the NFTs hold the knowledge reputation tokens on behalf of the member who owns the NFT. Those NFTs are non transferables which lock in the reputation with this member. The only way for a remember to gain those knowledge reputation tokens is by contributing through the research and activities required to advance the mission of the DAO.

This is a quintessential part of how the research DAO will be able to function without being dominated by one single entity. Naturally the individuals or group of researchers who contribute the most work we'll be able to get higher gains out of the digital assets (e.g., research grants, papers, reviews etc), that will pass through the collective. 
This is the actual desired outcome because it sets up a competitive landscape for the researchers in the DAO so that they can produce more research and gain more decision power in the DAO.

\subsection{Knowledge Contribution Token}

Knowledge Contribution tokens (KCT) are non transferable tokens. They can be only acquired by producing research work. New IP and data can be time-stamped and indisputably filed as NFTs on the blockchain as proof-of-knowledge for firmly claiming author-, and ownership, possibly backed by blockchain-based (self-sovereign) identity management (SSI). This means that the only benefit of gaining KCT is to participate in the DAO governance. They function like governance tokens except that they can not be traded. They represent a concrete proof of research contribution, thus we consider them proof of research.

Blockchain-enabled token economy may efficiently and transparently incentivize and coordinate an integrative and community-inclusive participatory approach to fuel crowdsourcing of collective intelligence [35]. The gaming aspect of staking the KCTs during the voting process ensures that the member is voting their conventions to the best of the entire DAO. They stand to lose those hard earned KCTs if they are voting in the wrong direction.

\subsection{Weighted NFTs}

An important consideration here is that we would need to set up some weights allowing different individuals to start from the same point. Weighted NFT's is an approach to assure that individuals in areas that do not have a lot of research facilities going through we'll be able to gain a voice in how the DAO is operated.

For example, members belonging to the same institution will have a reduced weight when voting and members of different institutions will have a slightly elevated weight.

\section{Conclusions}

Research is by far the most valuable aspect of human civilizations. Throughout human history, those civilizations capable of creating, using and passing on knowledge ended up with a lasting impact on the entire planet. Yet only if it is publicly available and accessible. While much scientific research is publicly funded or in the public interest, it is increasingly not a public good, available to the public, nor advancing the knowledge of humankind publicly. This is a global problem fueled by the increasing centralization of scientific research and knowledge in our academies and foundations; and the private commodification of research activities and outputs.

Decentralized Autonomous Organizations (DAO) represent the next evolution in global organizational governance. They are on the rise, and it is an exciting time for research scholars globally and organizational and technology scholars in particular, to address this emerging phenomenon with new theory and solid empirical research for a global research community.

We have presented a conceptual framework for adopting the best of both worlds of a global research community and of DAOs in creating the Global Research DAO (GR-DAO). We hope to be able to adopt this model and realize it in our next efforts.

\section{ACKNOWLEDGEMENTS}

The authors would like to thank the DEVxDAO and Learning Economy Foundation (LEF) for ongoing discussions about DAO solutions for the global research community. 


\section{REFERENCES}

[1] OECD, (2007) OECD Glossary of Statistical Terms - Research and development UNESCO Definition, Accessed from: stats.oecd.org. Archived from the original on 19 February 2007, Retrieved 24 September 2021.

[2] Kitcher, P, (2001) Science, truth, and democracy, New York: Oxford.

[3] Habermas, J, (1971) Knowledge and Human Interest, Beacon Press, Boston.

[4] Sintonen, M, (1990) "Basic and Applied Sciences-Can the Distinction (Still) Be Drawn?" Science Studies 3:2 (1990), 23-31.

[5] Krimsky, S, (2003). Science in the private interest. Lanham, MD: Rowman and Littlefield.

[6] Mody, C. C. M, (2006) "Corporations, universities, and instrumental communities: Commercializing probe microscopy, 1981-1996,” Technology and Culture, Vol. 47, No. 1, pp 5680 .

[7] Montgomery, K., and A. L, Oliver. (2009) "Shifts in guidelines for ethical scientific conduct: How public and private organizations create and change norms of research integrity" Social Studies of Science, Vol. 39, No. 1, pp 137-55.

[8] Tilak, J.B.G, (2008) "Higher education: a public good or a commodity for trade?" Prospects, Vol. 38, pp 449-466.

[9] Radder, Hans, (2010) "Chapter 1: The Commodification of Academic Research." In Hans Radder (ed.), The Commodification of Academic Research: Analyses Assessments, Alternatives, University of Pittsburgh Press.

[10] Calıskan, Koray and Callon, Michel, (2009) "Economization, part 1: shifting attention from the economy towards processes of economization" Economy and Society, Vol. 38, No. 3, pp 369-398.

[11] Calıskan, Koray and Callon, Michel, (2010) "Economization, part 2: A research programme for the study of markets" Economy and Society, Vol. 39, No. 1, pp. 1-32.

[12] Fairclough, Norman, (1993) "Critical Discourse Analysis and the Marketization of Public Discourse: The Universities" Discourse and Society, Vol. 4, No. 2, pp 133-168.

[13] Etzkowitz, H, (1998) "The norms of entrepreneurial science: Cognitive effects of the new university-industry linkages" Research Policy, Vol. 27, No. 8, pp 823-33.

[14] Kleinman, Daniel Lee (2010) "The Commercialization of Academic Culture and the Future of the University" In Hans Radder (ed.), The Commodification of Academic Research: Analyses Assessments, Alternatives, University of Pittsburgh Press.

[15] Alderman, G, (2009) "Higher education in the United Kingdom since 1945", Times Higher Education,

$30 \mathrm{July,} \mathrm{available} \mathrm{fromhttps://www.timeshighereducation.com/books/higher-education-in-theunited-}$ kingdom-since-1945/407560.paper?storycode=407560 [accessed January 2015]

[16] Dearlove, J, (1997) "The academic labour process: from collegiality and professionalism to managerialism and proletarianisation?" Higher Education Review, Vol. 30, pp 56-75.

[17] AGB, (1996) "Renewing the Academic Presidency: Stronger Leadership for Tougher Times", Association of Governing Boards of Universities and Colleges, Washington DC.

[18] Ben R. Martin, (2016) What's happening to our universities?, Prometheus, 34:1, 7-24.

[19] Burns, T. and Stalker, M, (1961) The Management of Innovation, Tavistock Publications, London.

[20] Khandwalla, P, (1977) The Design of Organizations, Harcourt Brace Jovanovich, New York, NY.

[21] Deal, T. and Kennedy, A, (1982) Corporate Culture, Addison-Wesley, Reading MA.

[22] Dewar, R. and Werbel, J, (1979) "Universalistic and contingency predictions of employee satisfaction and conflict", Administrative Science Quarterly, Vol. 24, pp 426-48.

[23] Schminke, M., Ambrose, M. \& Cropanzano, R, (2000) "The effect of organizational structure on perceptions of procedural fairness", Journal of Applied Psychology, Vol. 85, pp 294-304.

[24] Damanpour, F, (1991) "Organizational Innovation: A Meta-Analysis of Effects of Determinants and Moderators” The Academy of Management Journal, Vol. 34, No. 3 pp 555-590.

[25] Buranyi S, (2017). Is the staggeringly profitable business of scientific publishing bad for science? The Guardian Date: 27.6.2017. Accessed 25.9.2021.

[26] van Valkenburgh P, Dietz J, De Filippi P, Shadab H, Xethalis G, Bollier D (2015), "Distributed collaborative organisations: distributed networks and regulatory frameworks" Harvard Working Paper, Accessed 01 Aug 2016.

[27] Dietz J, Xethalis G, De Filippi P, Hazard J (2016), "Model distributed collaborative organizations" Stanford Working Group, Accessed 01 Aug 2016. 
[28] Nakamoto S, (2008) Bitcoin: a peer-to-peer electronic cash system. New York.

[29] Hsieh, YY., Vergne, JP., Anderson, P. et al., (2018) "Bitcoin and the rise of decentralized autonomous organizations" Journal of Organizational Design, Vol. 7, No. 14.

[30] McGregor-Lowndes, I. (2019). "The rise of the DAO disrupting 400 years of corporate structure" The Proctor, Vol. 39, No. 1, pp 32-33.

[31] Molinero, X., \& Riquelme, F. (2021) "Influence decision models: From cooperative game theory to social network analysis" Computer Science Review, Vol. 39.

[32] Molinero, X., Riquelme, F., \& Serna, M, (2015) "Cooperation through social influence.” European Journal of Operational Research, Vol. 242, No. 3.

[33] ElMessiry, M., ElMessiry, A., \& ElMessiry, M. (2019). "Dual token blockchain economy framework" In the International Conference on Blockchain, Springer, Cham. pp. 157-170.

[34] Popescu, A. (2021). "Non-Fungible Tokens (NFT) - Innovation beyond the craze." 5th International Conference on Innovation in Business, Economics \& Marketing research (IBEM-2021) Proceedings of Engineering \& Technology - PET - Vol 66. pp. 26-30.

[35] Ducrée, J. (2020). "Research - A blockchain of knowledge?" Blockchain: Research and Applications, Vol 1. (1-2).

\section{AUTHORS}

\section{Kelly, L. Page, Ph.D.}

A social design ethnographer, social and digital innovator, and learning entrepreneur committed to developing truly social cultures, people, and organizations with emerging and social technology. Kelly has a Ph.D. in the Psychology of Web (Hypermedia) Knowledge from UNSW and an obsession with learning innovation and digital social storytelling. She has over 18 years of experience working at the intersection of social innovation, social design, and learning of mediated social experiences for Startups, Universities, Schools, and School Districts, to Fortune 500 companies. She believes that innovative and entrepreneurial thinking is at the heart of creating truly social cultures, organizations, and leaders.

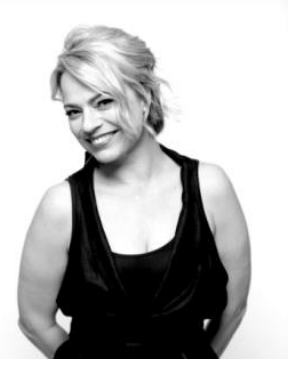

Her work has been published in leading peer-reviewed academic education, technology, and business journals, such as Journal of Business Research, Studies in Higher Education, Computers in Human Behavior,

International Journal of Interactive Marketing, International Journal of Human-Computer Studies, Psychology\& Marketing, Behavior \& Information Technology .... and been featured in The New York Times, Fast Company, Wall Street Journal. Nominated for an Edison Innovation Award, her work has received awards from IDMA and a BIMA for Best in British Digital.

\section{Adel Elmessiry, Ph.D.}

Tech entrepreneur, published expert on AI and Blockchain with 20+ years Healthcare, Mentor, Advisors \& Speaker. Adel is a serial entrepreneur with three successful technology companies taken from inception to acquisition. He has a proven executive experience with a solid track record that includes over 10 years at HealthStream and 7 years at InVivoLink/ HealthTrust. Academically, he is holding a Ph.D. in Computer Science at NCSU Natural Language Processing. He serves as the president chief technology officer for AlphaFin, a Draper Goren Holm portfolio company. Together we are on a mission to build the next financial technology ecosystem that will empower the global economy.

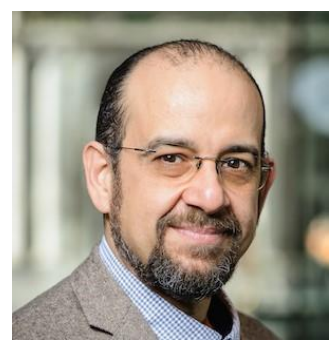

(C) 2021 By AIRCC Publishing Corporation. This article is published under the Creative Commons Attribution (CC BY) license. 